\title{
Coherence times of precisely depth controlled NV centers in diamond
}

\author{
Junfeng Wang, ${ }_{1}$ Wenlong Zhang, ${ }_{1}$ Jian Zhang, ${ }^{1}$ Jie You, ${ }^{2}$ Yan Li,${ }^{2}$ Guoping Guo, ${ }^{2}$ \\ Fupan Feng, ${ }^{1}$ Xuerui Song, ${ }^{1}$ Liren Lou, ${ }^{1}$ Wei Zhu, ${ }^{1}$ and Guanzhong Wang ${ }^{1,}$, \\ ${ }^{1}$ Hefei National Laboratory for Physical Science at Microscale, and Department of Physics, \\ University of Science and Technology of China, Hefei, Anhui, 230026, P. R. China \\ ${ }^{2}$ Key lab of Quantum Information, CAS, University of Science and Technology of China, Hefei, Anhui, 230026, P. R. China
}

We investigated the depth dependence of coherence times of nitrogen-vacancy (NV) centers through precisely depth controlling by a moderately oxidative at $580{ }^{\circ} \mathrm{C}$ in air. By successive nanoscale etching, NV centers could be brought close to the diamond surface step by step, which enable us to trace the evolution of the number of NV centers remained in the chip and to study the depth dependence of coherence times of NV centers with the diamond etching. Our results showed that the coherence times of NV centers declined rapidly with the depth reduction in their last about $22 \mathrm{~nm}$ before they finally disappeared, revealing a critical depth for the influence of rapid fluctuating surface spin bath. By monitoring the coherence time variation with depth, we could make a shallow NV center with long coherence time for detecting external spins with high sensitivity.

In recent years, shallow NV center has attracted increasing attention owing to its applications in nanoscale spin detection [1-7] and surface spin noise investigation [811]. Since the minimum detectable magnetic dipole moment scales as $\delta \mu \propto r^{3} / \sqrt{T_{2}}$ 9, 12, 13], where $\mathrm{r}$ is the $\mathrm{NV}$-target spin distance and $T_{2}$ is the coherence times of the NV center, the nanoscale control of the NV center depth and the investigation of depth dependence of NV center coherence time are important.

Recently, two methods, plasma etching [14] and oxidative etching [13, 15], have been developed to control the depth of the NV center. Cui et al. found that the conventional oxygen plasma etching had low damage to diamond [14]. For oxidative etching, Kim et al. demonstrated that the diamond could be etched at the temperature, ranging from $550{ }^{\circ} \mathrm{C}$ to $620{ }^{\circ} \mathrm{C}$, in pure oxygen gas [13]. Loretz et al. reported that the oxidative etching rate for diamond was about $10 \mathrm{~nm} / \mathrm{h}$ at $650{ }^{\circ} \mathrm{C}$ in air and by using this method they realized a 1.9-nm-deep NV center [15]. However, the etching rate was not short enough to precisely control the depth of NV center .

In this work, we performed oxidative etching in air on diamond at a reduced oxidative temperature $580{ }^{\circ} \mathrm{C}$, obtaining about $1.1 \mathrm{~nm} / \mathrm{h}$ etching rate. By successive nanoscale etching, NV centers could be brought close to the diamond surface step by step, which enable us to investigate the depth dependence of coherence times of $\mathrm{NV}$ centers. The coherence time of NV center declined rapidly with the depth reduction in their last about 22 $\mathrm{nm}$ before they finally disappeared, which was attributed to the influence of the rapid fluctuating surface spin bath. By monitoring the coherence time variation with depth, we could make a shallow NV center with long coherence time for detecting external spins with high sensitivity $[5$, 7, 15, 16].

A $2 \times 2 \times 0.5 \mathrm{~mm}^{3}(100)$ electronic grade diamond chip from Element Six $\left(\left[{ }^{13} \mathrm{C}\right]=1.1 \%,[\mathrm{~N}]<5 \mathrm{ppb}\right)$ was used for the experiments. At first, a 300-nm-thick polymethyl methacrylate (PMMA) layer was deposited on the dia-

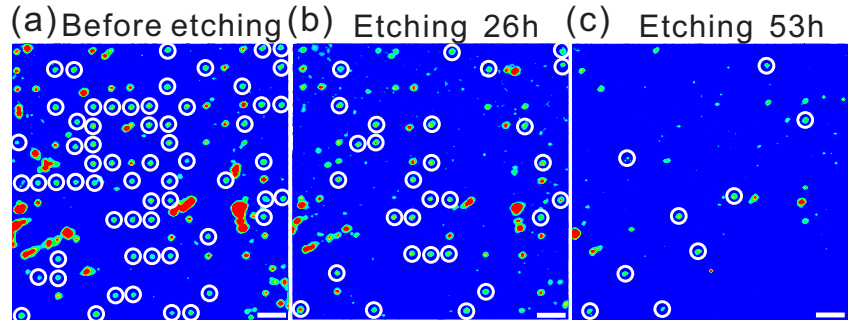

(d)

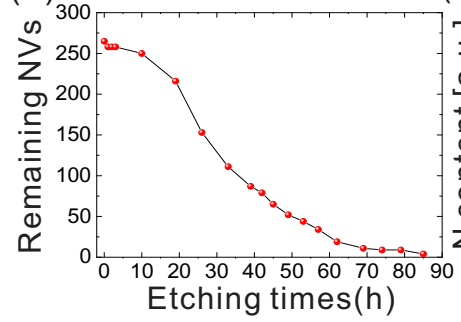

(e)
FIG. 1. Fluorescence images of the remaining NV centers in the diamond chip after different oxidative etching times. (a) Image of a representative area of the chip before oxidative etching. (b) and (c) The same area after oxidative etching 26 $\mathrm{h}$ and $53 \mathrm{~h}$, respectively. The circled single NV centers in the (a) ((b) and (c)) were used for tracing. All the scale bars in (a) ((b), (c)) are $5 \mu \mathrm{m}$. (d) The total number of the remaining NV centers in the selected area on the diamond chip after different oxidative etching times. (e) The SRIM simulations of the depth profile of the implanted nitrogen atoms at an energy of $30 \mathrm{keV}$.

mond chip surface [17, 18]. Then a series of $60 \mathrm{~nm}$ diameter apertures, as well as some 10- $\mu m$-wide strips used as position marks [18], were patterned on the PMMA layer using electron beam lithography. The NV centers in the chip were generated by implanting $60 \mathrm{keV}{ }^{14} N_{2}^{+}$ molecules with a fluence $0.55 \times 10^{11} 14 N_{2}^{+} / \mathrm{cm}^{2}$ through the apertures and strips on the PMMA layer. 17, 18]. After implantation, the sample was annealed at $1050{ }^{\circ} \mathrm{C}$ in a vacuum at $2 \times 10^{-5} \mathrm{~Pa}$ for $2 \mathrm{~h}$ to form long spin coherence time NV centers [19]. After oxidation at $430{ }^{\circ} \mathrm{C}$ 
(a)

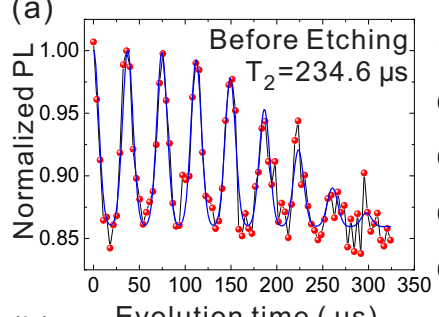

(b)

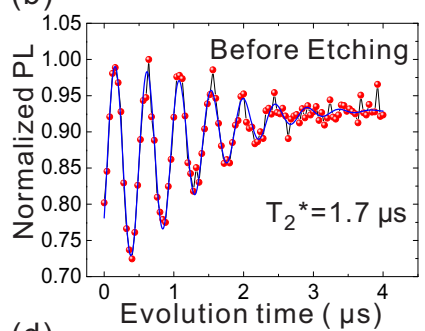

(d)

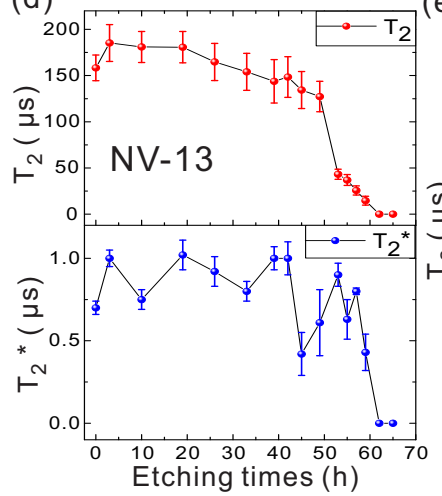

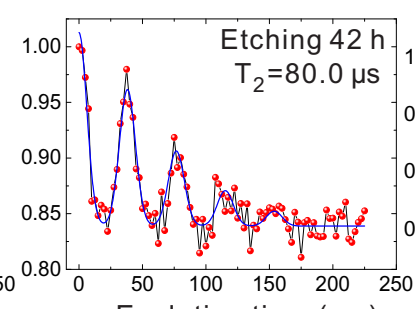

Evolution time ( $\mu s)$

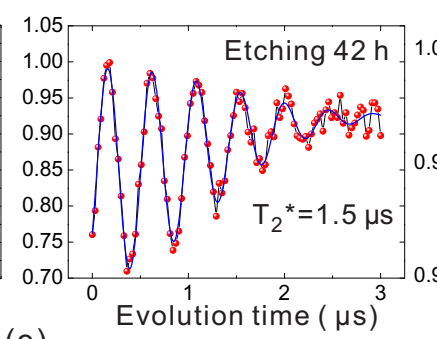

(e)

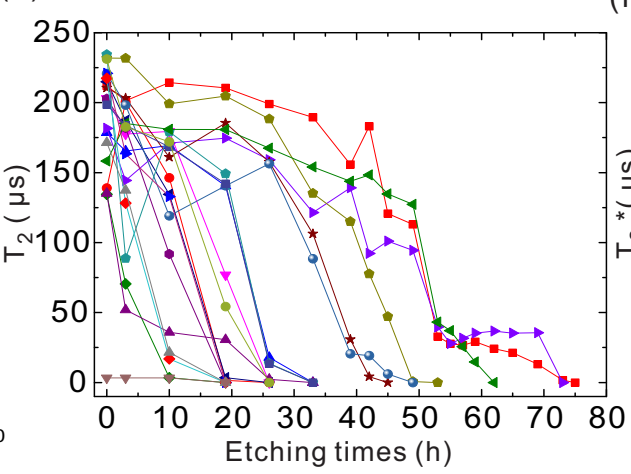

(c)

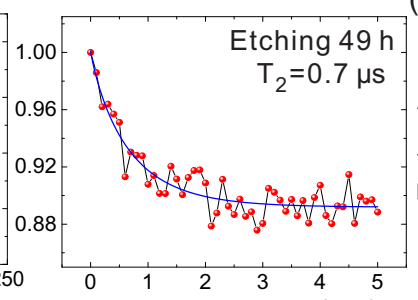

Evolution time ( $\mu \mathrm{s})$

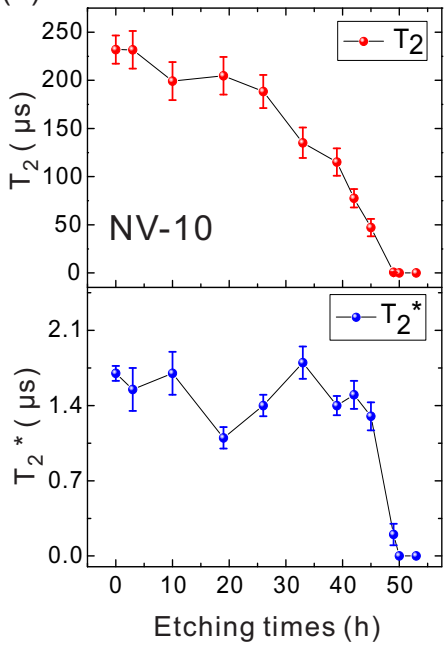

(f)

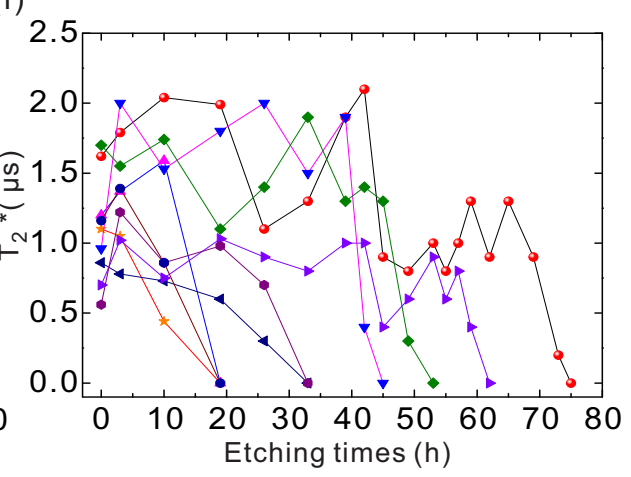

FIG. 2. Coherence times measurements of the NV centers after different oxidative etching times. (a) and (b) Spin echo and Ramsey measurements, respectively, of NV-10 after three different oxidative etching times. The blue lines were the fits of the $I_{P L}$. (c) and (d) The spin echo and Ramsey measurements of NV-10 and NV-13 after different oxidative etching times, respectively. (e) Coherence times vs. etching time derived from spin echo measurements for all the 20 single NV centers. (f) Coherence times vs. etching time derived from Ramsey measurements for 9 representative single NV centers.

in air for $2.5 \mathrm{~h}$, the sample was cleaned in a 1:1:1 boiling mixture of sulfuric, nitric, and perchloric acid at $200{ }^{\circ} \mathrm{C}$ for 2 hours.

The oxidative etching was performed in air on the diamond chip situated in a box furnace with its implanted side up. In order to have a slow etching rate, we used a lower oxidative temperature of $580{ }^{\circ} \mathrm{C}$. The diamond chip was put in the furnace when the furnace temperature had already stabilized at $580{ }^{\circ} \mathrm{C}$. The process for the furnace temperature being stabilized at $580{ }^{\circ} \mathrm{C}$ only took about 1 minute. Similarly, when the etching process was finished, we directly took the diamond chip out to the air for cool down. We traced the evolution of the number of single NV centers remaining in the chip with the etching time and investigated the relationship between the coherence times of the NV centers and the etching times. Figure 1(a) showed the fluorescence image of a representative area of the diamond chip before oxidative etching, with the traced single NV centers marked with circles in the figure. The larger bright specks in the image were
$\mathrm{NV}$ center clusters produced in the implantation due to imperfection of the PMMA templet layer deposited on the diamond chip [18]. After oxidative etching for $26 \mathrm{~h}$, the image of the same area showed less single NV centers and the bright specks of the NV center clusters also became less bright and smaller [Fig. 1(b)]. As shown in Fig. 1(c), after oxidative etching for $53 \mathrm{~h}$, there were only few single NV centers remained on the chip and the bright specks became even less bright and smaller. These results clearly demonstrated that the NV centers originally situated at different depths of the diamond chip had been removed successively by oxidative etching [13, 15].

We traced 265 single NV centers that were initially in the diamond chip. As shown in Fig. 1(d), the number of the remaining single NV centers in the chip reduced with the oxidative etching times. We found that when the etching time was less than $10 \mathrm{~h}$, the number of the remaining NV centers reduced very little. Then, when the etching times increased from $10 \mathrm{~h}$ to about $60 \mathrm{~h}$, the NV centers number reduced rapidly. After that, the number 

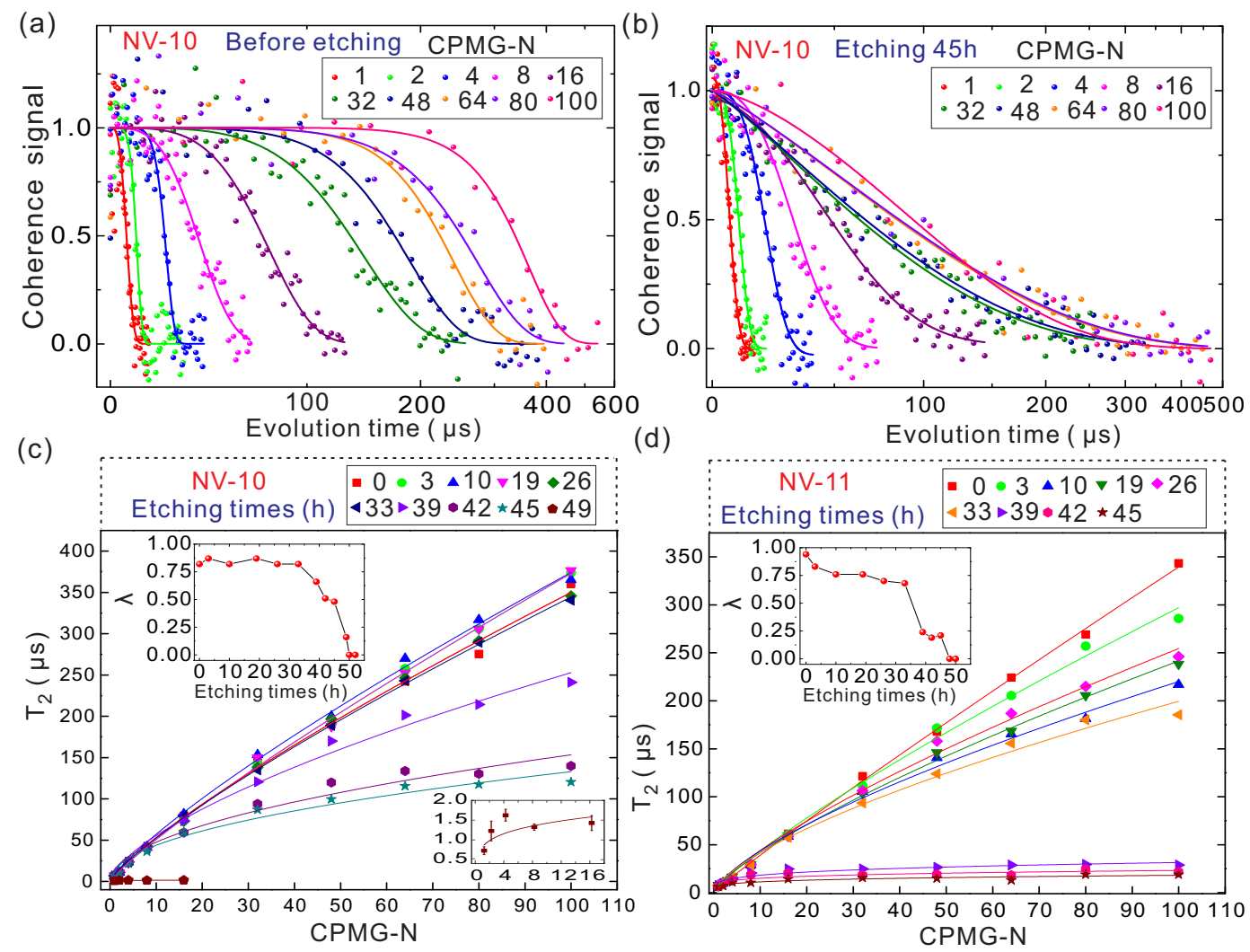

FIG. 3. Dynamical decoupling measurements after different oxidative etching times. (a) and (b) The coherence decay curves of NV-10 in CPMG-N with the $\pi$ pulse number $\mathrm{N}$ from 1 to 100 for the sample before etching and with etching $45 \mathrm{~h}$, respectively. The solid lines were the fits to the data using the exponentials $e^{-\left(t / T_{2}\right)^{n}}$, where $T_{2}$ is the coherence times. (c) The coherence times $T_{2}$ of the CPMG of NV-10 as functions of $\mathrm{N}$ for the chip etched for different times. The inset (bottom right) was the zoom of the CPMG-N ( $\mathrm{N}$ from 1 to 16) results for the chip after etching $49 \mathrm{~h}$. (d) The coherence times $T_{2}$ of the CPMG of NV-13 as functions of $\mathrm{N}$ after different oxidative etching times. The solid lines in the (c) and (d) were the fits to the data using the power-law $T_{2, C P M G-N} / T_{2, \text { echo }}=N^{\lambda}$ and the decoupling efficiency $\lambda$ as a function of the etching time for both cases were shown in the inset (top left) of the corresponding figures, respectively.

reduced slowly and finally almost all the NV centers finally disappeared after $85 \mathrm{~h}$ etching. The results showed that the number of the remaining single NV centers reduced about $70 \%$ after etching $42 \mathrm{~h}$. By comparing the above results with the SRIM simulations for an implantation nitrogen atom energy of $30 \mathrm{keV}$ [Fig. 1(e)], we found that the etching depth corresponding to $42 \mathrm{~h}$ etching was $46 \mathrm{~nm}$. Therefore, we estimated that the etching rate was about $1.1 \mathrm{~nm} / \mathrm{h}$ at $580 \mathrm{C}$ in air.

The slow etching rate made it possible to control the depth of the NV center at nanoscale. Thus, we could precisely trace the evolution of the coherence time of a single NV center with its depth, which reflected the variation in its local environment, from the bulk ${ }^{13} \mathrm{C}$ spin bath to the surface spin bath. A set of randomly selected 20 single NV centers [named NV-1 through 20] with their axes parallel to the external magnetic field $(47 \mathrm{G})$ was used for the tracing. We performed spin echo measurements for all the 20 single NV centers before the chip etching, and found that the $T_{2}$ of $19 \mathrm{NV}$ centers among them were between 134 and $234 \mu s$, and the other one was $3.3 \mu \mathrm{s}$. The mean value of $T_{2}$ of the 19 single NV centers was about $194 \mu s$, which was comparable to that of the native NV center inside electronic grade diamond 20], implying that the NVs were initially deep inside the chip and the initial major spin impurities were ${ }^{13} C$.

Then, we measured the coherence times of the $20 \mathrm{NV}$ centers after successively etching for various times step by step. Fig. 2(a) showed three representative results of the spin echo measurements for NV-10 (which just disappeared after etching $50 \mathrm{~h}$ ), after different oxidative etching times. In the first two figures, we found that the photoluminescence (PL) profile collapsed and revived at ${ }^{13} \mathrm{C}$ Larmor frequency (about $50.3 \mathrm{kHz}$ ), which was due to the incoherent precession of the ${ }^{13} \mathrm{C}$ nuclei spins in our natural ${ }^{13} \mathrm{C}$ abundance samples 21]. It was found that for the sample before etching, the $T_{2}$ of NV-10 was 234.6 $\mu s$. And, after an etching of $42 \mathrm{~h}$, the $T_{2}$ declined to 80 $\mu s$. However, when the etching time reached $49 \mathrm{~h}$, its PL intensity was not stable and was reduced to about half of the initial value, indicating that the center had been very shallow and its charge state had become unstable 
[22, 23]. (The similar phenomenon was also observed for other NV centers, when they were shallow enough by long time etching.) Moreover, its $T_{2}$ was reduced to only about $0.7 \mu \mathrm{s}$. The above results revealed that the NV center became very shallow and suffered a strong influence of the rapid fluctuating surface spin bath 8 10, 24]. The $T_{2}$ of NV-10 in the sample etched for various times were presented in Fig. 2(c) top. This etching time dependence can be converted to the depth dependence as the depth varied with the etching time. As shown from the figure, for short etching time (less than about $30 \mathrm{~h}$ ), the $T_{2}$ decreased slowly with the etching time, then declined rapidly in its last about $20 \mathrm{~h}$ etching before it finally disappeared (i.e. when its depth was less than about $22 \mathrm{~nm}$ ). Finally, when the etching time was longer than 50 h, NV-10 was finally disappeared.

Three representative Ramsey measurements for NV-10 were also shown in Fig. 2(b), and its $T_{2}^{*}$ for the sample after various etching times were summarized in Fig. 2(c) bottom. We could see that the $T_{2}^{*}$ had a similar evolution to the $T_{2}$. As another example, Figure 2(d) gave the results of the coherence time measurements for NV13 (just disappeared after etching $62 \mathrm{~h}$ ). It is clear that the coherence time decreased very slowly with the etching time in the initial $\sim 40 \mathrm{~h}$, while declined rapidly in the last $\sim 20$ h etching before it finally disappeared (i.e. when its depth was less than about $22 \mathrm{~nm}$ ). The evolution were similar to that of NV-10. Fig. 2(e) summarized the dependences of $T_{2}$ with the etching time for all the $20 \mathrm{NV}$ centers. We found that there was a similar dependence for all these NV centers: the $T_{2}$ of each NV centers decreased rapidly when the etching time was about $20 \mathrm{~h}$ before the centers finally disappeared (i.e. when their depth became less than about $22 \mathrm{~nm}$ ). The results of Ramsey measurements of the 9 representative single $\mathrm{NV}$ centers after different oxidative etching times were shown in Fig. 2(f), which exhibited a similar variation as that of their $T_{2}$ in Fig. 2(e). The results suggested that, when the depth decreased to about $22 \mathrm{~nm}$, rapidly fluctuating surface spins, compared with the slow bulk ${ }^{13} C$ spin bath, began to play an important role in $\mathrm{NV}$ decoherence, leading to a rapid decreasing in coherence time with a further decrease in centers depth, which was consistent with the results of the delta doping NV centers [9].

Dynamical decoupling was important in NV center based high sensitivity magnetic field [25] and temperature [18, 26] sensing. In particular, the shallow NV centers, combined with dynamical decoupling, could also be used to detect external spins [3, 5-7] and investigate the surface spin noise 9 11]. In view of this, we performed CPMG measurements on three arbitrarily selected NV centers (NV-1, 10, 11) on the chip after different oxidative etching times. The results were shown in Fig. 3. As shown in Fig. 3(a), before etching, the $T_{2}$ of the CPMG$\mathrm{N}$ of NV-10 were always increasing with the $\pi$ pulse num- ber $\mathrm{N}$, in particular, the $T_{2}$ of the CPMG-100 was about $360.4 \mu \mathrm{s}$, about 52 times longer than the value $6.9 \mu \mathrm{s}$ for the spin echo. However, in Fig. 3(b), after etching for $45 \mathrm{~h}$, almost all the $T_{2}$ of the CPMG-N were declined, in particular, the $T_{2}$ of the CPMG-100 was about $120.4 \mu \mathrm{s}$, which was only about one third of the value before etching. The $T_{2}$ of the CPMG-N as a function of $\mathrm{N}$ for $\mathrm{NV}-10$ with the chip etched for various times were summarized in Fig. 3(c). The decoupling efficiencies $\lambda$ [9] varied with the etching time were shown in the inset (top left) of Fig. 3(c). When the etching time was less than about $30 \mathrm{~h}$, the $T_{2}$ of the CPMG were decreased very slowly, and the decoupling efficiencies $\lambda$ was nearly a constant of about 0.82 . This was consistent to the fact that the center, for sample etched for $30 \mathrm{~h}$, was still inside the chip and the main spin bath was a bulk ${ }^{13} C$ bath [20]. Then, they both declined rapidly when its depth decreasing in its last about $20 \mathrm{~h}$ etching before it finally disappeared (i.e. when its depth was less than about $22 \mathrm{~nm}$ ), which was similar to the results in Fig. 2(c). The similar phenomena were observed for NV-11 (just disappeared after etching 48 h) [Fig. 3(d)] and NV-1 (just disappeared after etching $75 \mathrm{~h}$, and its data was not shown in this paper). The results were consistent with the fact that when the depth of the NV centers decreased, they would have a higher coupling strength with surface spin bath that had a fast fluctuation rate [9, 10].

In summary, we investigated the depth dependence of coherence times of NV centers in diamond with the depth controlled using oxidative etching. We used a lower oxidative temperature of $580{ }^{\circ} \mathrm{C}$ in air to reduce the etching rate to about $1.1 \mathrm{~nm} / \mathrm{h}$. Thus, we can more precisely control the depth of the centers. On the basis of this, we performed the spin echo and Ramsey and CPMG measurements on the NV centers, of which the depth was decreased by successive oxidative etching, and found that the coherence times of NV centers declined rapidly with the depth reduction in their last about $22 \mathrm{~nm}$ etching before they finally disappeared, revealing a critical depth for the influence of rapid fluctuating surface spin bath, which was consistent with the results of the delta doping NV centers. [9]. The obtained results showed how the surface spin influenced the coherence behavior of NV centers of different depths, paving the way for investigating surface spins. Particularly, the slow etching method make it possible to control the depth of the NV center at nanoscale, which can be used to study the stability of the very shallow NV centers [24, 25, 27], to investigate the surface spin noise [8 11], and for high sensitivity (even for single electron and nuclear) nanoscale spin detection [5, 7, 15, 16]. Moreover, it can also be used to control the depth of the NV center in the diamond tip equipped to the atomic force microscope, which can be used in high sensitivity nanoscale magnetic imaging of the spins [28].

This work was supported by the National Basic Research Program of China (2013CB921800, 
2011CB921400) and the Natural Science Foundation of China (Grants No. 11374280 and No. 50772110).

* gzwang@ustc.edu.cn

[1] B. Grotz, J. Beck, P. Neumann, B. Naydenov, R. Reuter, F. Reinhard, F. Jelezko, J. Wrachtrup, D. Schweinfurth, B. Sarkar, and P. Hemmer, New J. Phys. 13, 055004 (2011).

[2] H. J. Mamin, M. H. Sherwood, and D. Rugar, Phys. Rev. B 86, 195422 (2012).

[3] T. Staudacher, F. Shi, S. Pezzagna, J. Meijer, J. Du, C. A. Meriles, F. Reinhard, and J. Wrachtrup, Science 339, 561 (2013).

[4] H. J. Mamin, M. Kim, M. H. Sherwood, C. T. Rettner, K. Ohno, D. D. Awschalom, and D. Rugar, Science 339, 557 (2013).

[5] C. Müller, X. Kong, J.-M. Cai, K. Melentijević, A. Stacey, M. Markham, D. Twitchen, J. Isoya, S. Pezzagna, J. Meijer, J. F. Du, M. B. Plenio, B. Naydenov, L. P. McGuinness, and F. Jelezko, Nat. Commun. 5, 4703 (2014).

[6] S. J. DeVience, L. M. Pham, I. Lovchinsky, A. O. Sushkov, N. Bar-Gill, C. Belthangady, F. Casola, M. Corbett, H. Zhang, M. Lukin, H.K. Park, A.Yacoby, and R. L. Walsworth, Nat. Nano. 10, 129 (2015).

[7] F. Z. Shi, Q. Zhang, P. F. Wang, H. B. Sun, J. R. Wang, X. Rong, M. Chen, C. Y. Ju, F. Reinhard, H. W. Chen, J. Wrachtrup, J. F. Wang, and J. F. Du, Science 347, 1135 (2015).

[8] T. Rosskopf, A. Dussaux, K. Ohashi, M. Loretz, R. Schirhagl, H. Watanabe, S. Shikata, K. M. Itoh, and C. L. Degen, Phys. Rev. Lett. 112, 147602 (2014).

[9] B. A. Myers, M. C. Dartiailh, K. Ohno, A. Das, D. D. Awschalom, and A. C. B. Jayich, Phys. Rev. Lett. 113, 027602 (2014).

[10] Y. Romach, C. Muller, T. Unden, L. J. Rogers, T. Isoda, K. M. Itoh, M. Markham, A. Stacey, J. Meijer, S. Pezzagna, B. Naydenov, L. P. McGuinness, N. Bar-Gill, and F. Jelezko, Phys. Rev. Lett. 114, 017601 (2015).

[11] L. Luan, M. S. Grinolds, S.K. Hong, P. Maletinsky, R. L. Walsworth, and A. Yacoby, Sci. Rep. 5, 8119 (2015).

[12] J. R. Maze, P. L. Stanwix, J. S. Hodges, S. Hong, J. M. Taylor, P. Cappellaro, L. Jiang, M. V. Gurudev Dutt, E. Togan, A. S. Zibrov, A. Yacoby, R. L. Walsworth, and M. D. Lukin, Nature (London) 455, 644 (2008).

[13] M. Kim, H. J. Mamin, M. H. Sherwood, C. T. Rettner, J. Frommer, and D. Rugar, Appl. Phys. Lett. 105, 042406
(2014).

[14] S. Y. Cui, A. S. Greenspon, K. Ohno, B. A. Myers, A. C. Bleszynski Jayich, D. D. Awschalom, and E. L. Hu, Nano Lett. 15, 2887 (2015).

[15] M. Loretz, S. Pezzagna, J. Meijer, and C. L. Degen, Appl. Phys. Lett. 104, 033102 (2014).

[16] A. O. Sushkov, I. Lovchinsky, N. Chisholm, R. L. Walsworth, H. Park, and M. D. Lukin, Phys. Rev. Lett. 113, 197601 (2014).

[17] P. Spinicelli, A. Dréau, L. Rondin, F. Silva, J. Achard, S. Xavier, S. Bansropun, T. Debuisschert, S. Pezzagna, J. Meijer, V. Jacques, and J.-F. Roch, New J. Phys. 13, 025014 (2011).

[18] J. F. Wang, F. P. Feng, J. Zhang, J. H. Chen, Z. C. Zheng, L. P. Guo, W. L. Zhang, X. R. Song, G. P. Guo, L. L. Fan, C.W. Zou, L. R. Lou, W. Zhu, and G. Z Wang, Phys. Rev. B 91, 155404 (2015)

[19] T. Yamamoto, T. Umeda, K. Watanabe, S. Onoda, M. L. Markham, D. J. Twitchen, B. Naydenov, L. P. McGuinness, T. Teraji, S. Koizumi, F. Dolde, H. Fedder, J. Honert, J. Wrachtrup, T. Ohshima, F. Jelezko, and J. Isoya, Phys. Rev. B 88, 075206 (2013).

[20] C. A. Ryan, J. S. Hodges, and D. G. Cory, Phys. Rev. Lett. 105, 200402 (2010).

[21] L. Childress, M. V. Gurudev Dutt, J. M. Taylor, A. S. Zibrov, F. Jelezko, J. Wrachtrup, P. R. Hemmer, and M. D. Lukin, Science 314, 281 (2006).

[22] R. Schirhagl, K. Chang, M. Loretz, and C. L. Degen, Annu. Rev. Phys. Chem. 65, 83 (2014).

[23] C. Bradac, T. Gaebel, N. Naidoo, M. J. Sellars, J. Twamley, L. J. Brown, A. S. Barnard, T. Plakhotnik, A. V. Zvyagin, and J. R. Rabeau, Nat. Nanotechnol. 5, 345 (2010).

[24] B. K. Ofori-Okai, S. Pezzagna, K. Chang, M. Loretz, R. Schirhagl, Y. Tao, B. A. Moores, K. Groot-Berning, J. Meijer, and C. L. Degen, Phys. Rev. B 86, 081406 (R) (2012).

[25] L. M. Pham, N. Bar-Gill, C. Belthangady, D. Le Sage, P. Cappellaro, M. D. Lukin, A. Yacoby, and R. L. Walsworth, Phys. Rev. B 86, 045214 (2012).

[26] D. M. Toyli, C. F. de las Casas, D. J. Christle, V. V. Dobrovitski, and D. D. Awschalom, Proc. Natl. Acad. Sci. USA 110, 8417 (2013).

[27] I. I. Vlasov, A. A. Shiryaev, T. Rendler, S. Steinert, S.-Y. Lee, D. Antonov, M. Vörös, F. Jelezko, A. V. Fisenko, L. F. Semjonova et al., Nat. Nanotechnol. 9, 54 (2013).

[28] M. S. Grinolds, S. Hong, P. Maletinsky, L. Luan, M. D. Lukin, R. L. Walsworth, and A. Yacoby, Nat. Phys. 9, 215 (2014). 